International Journal of Pure and Applied Mathematics

Volume 104 No. 4 2015, 577-584

ISSN: 1311-8080 (printed version); ISSN: 1314-3395 (on-line version)

url: http://www.ijpam.eu

doi: http://dx.doi.org/10.12732/ijpam.v104i4.9

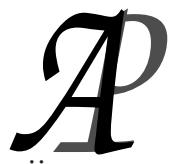

ijpam.eu

\title{
ON SUBMANIFOLDS OF GENERALIZED RECURRENT MANIFOLDS
}

\author{
Jaeman Kim \\ Department of Mathematics Education \\ Kangwon National University \\ Chunchon 200-701, Kangwon Do, KOREA
}

\begin{abstract}
In this paper we investigate some properties of submanifolds of generalized recurrent manifold (briefly, $G R M_{n}$ ). Firstly, we show that a totally geodesic hypersurface of $G R M_{n}$ is a $G R M_{n-1}$. Secondly, if $G R M_{n}$ is a Riemannian product manifold, then either one decomposition manifold is locally symmetric or the other decomposition manifold is a space of constant curvature. Thirdly, a Riemannian product manifold of a space of constant curvature with itself is a $G R M_{n}$.
\end{abstract}

AMS Subject Classification: 53A55, 53B20

Key Words: totally umbilic hypersurface, totally geodesic hypersurface, generalized recurrent manifold, Riemannian product manifold, associated 1forms, a space of constant curvature

\section{Introduction}

As a generalization of the notion of a space of constant curvature, the notion of (locally) symmetric manifolds was introduced by Cartan [4] who obtained a classification of such manifolds. During the last six decades, the notion of

Received: July 22, 2015

(c) 2015 Academic Publications, Ltd. url: www.acadpubl.eu 
locally symmetric manifolds was weakened by many authors in various ways to different extent such as recurrent manifolds [10], conformally recurrent manifolds [1], 2-recurrent manifolds [7], Ricci recurrent manifolds [9], concircularly recurrent manifolds [8] and projectively recurrent manifolds [2]. As an extending notion of recurrent manifolds, the notion of generalized recurrent manifolds was introduced by Dubey [6] and this manifold has received a great deal of attention. In [3] Arslan et al studied such a manifold in considerable detail. A Riemannian manifold $\left(M^{n}, g\right)(n \geq 3)$ is said to be generalized recurrent if its curvature tensor $R$ of type $(0,4)$ satisfies the condition

$$
\left(\nabla_{X} R\right)(Y, Z, V, W)=A(X) R(Y, Z, V, W)+B(X)(g \bullet g)(Y, Z, V, W),
$$

where $\nabla$ denotes the Levi-Civita connection and $A, B$ are the associated 1forms. Here the symbol $\bullet$ is the Nomizu-Kulkarni product of symmetric $(0,2)$ tensors generating a curvature type tensor:

$$
\begin{aligned}
(h \bullet k)(X, Y, Z, W)=h(X, Z) k(Y, W) & +h(Y, W) k(X, Z) \\
& -h(X, W) k(Y, Z)-h(Y, Z) k(X, W) .
\end{aligned}
$$

From now on, in this paper, an $n$-dimensional generalized recurrent manifold is denoted by $G R M_{n}$. In particular, if $B=0$ in (1.1), then the manifold reduces to a recurrent manifold. The purpose of this paper is to investigate some properties of a Riemannian product $G R M_{n}$ and hypersurfaces of $G R M_{n}$.

\section{Preliminaries}

Let $\left(M^{n}, g\right)$ be an $n$-dimensional Riemannian manifold covered by a system of coordinate neighborhoods $\left\{U ; y^{\alpha}\right\}$ and $\left(\bar{M}^{n-1}, \bar{g}\right)$ a hypersurface of $\left(M^{n}, g\right)$ covered by a system of coordinate neighborhoods $\left\{V ; x^{i}\right\}$. Let $y^{\alpha}=y^{\alpha}\left(x^{i}\right)$ be the parametric representation of the hypersurface $\bar{M}^{n-1}$ in $M^{n}$, where Greek indices take the values $1,2, \ldots, n$ and Latin indices take the values $1,2, \ldots, n-1$. Then we have

$$
\bar{g}_{i j}=g_{\alpha \beta} \frac{\partial y^{\alpha}}{\partial x^{i}} \frac{\partial y^{\beta}}{\partial x^{j}} .
$$

Here we adopt the Einstein convention, that is, when an index variable appears once in an upper and once in a lower position in a term, it implies summation of that term over all the values of the index. Let $N^{\alpha}$ be a local unit normal to 
$\left(\bar{M}^{n-1}, \bar{g}\right)$. Then we have the relations

$$
g_{\alpha \beta} N^{\alpha} \frac{\partial y^{\beta}}{\partial x^{j}}=0, g_{\alpha \beta} N^{\alpha} N^{\beta}=1, g^{\alpha \beta}=\bar{g}^{i j} \frac{\partial y^{\alpha}}{\partial x^{i}} \frac{\partial y^{\beta}}{\partial x^{j}}+N^{\alpha} N^{\beta} .
$$

The structure equations of Gauss and Codazzi for a hypersurface $\left(\bar{M}^{n-1}, \bar{g}\right)$ of $\left(M^{n}, g\right)$ can be respectively written as

$$
\begin{gathered}
\bar{R}_{i j k l}=R_{\alpha \beta \gamma \delta} \frac{\partial y^{\alpha}}{\partial x^{i}} \frac{\partial y^{\beta}}{\partial x^{j}} \frac{\partial y^{\gamma}}{\partial x^{k}} \frac{\partial y^{\delta}}{\partial x^{l}}+\bar{\omega}_{i l} \bar{\omega}_{j k}-\bar{\omega}_{i k} \bar{\omega}_{j l}, \\
R_{\alpha \beta \gamma \delta} \frac{\partial y^{\alpha}}{\partial x^{i}} \frac{\partial y^{\beta}}{\partial x^{j}} \frac{\partial y^{\gamma}}{\partial x^{k}} N^{\delta}=\bar{\omega}_{j k ; i}-\bar{\omega}_{i k ; j},
\end{gathered}
$$

where $\bar{R}_{i j k l}$ and $R_{\alpha \beta \gamma \delta}$ are the curvature tensors of $\left(\bar{M}^{n-1}, \bar{g}\right)$ and $\left(M^{n}, g\right)$ respectively, and $\bar{\omega}_{i j}$ is the second fundamental form of $\left(\bar{M}^{n-1}, \bar{g}\right)$.

The hypersurface $\left(\bar{M}^{n-1}, \bar{g}\right)$ is said to be a totally umbilic hypersurface of $\left(M^{n}, g\right)[5]$ if its second fundamental form $\bar{\omega}_{i j}$ satisfies

$$
\bar{\omega}_{i j}=H \bar{g}_{i j},\left(\frac{\partial y^{\alpha}}{\partial x^{i}}\right)_{; j}=\bar{g}_{i j} H N^{\alpha},
$$

where $H$ denotes the mean curvature of $\left(\bar{M}^{n-1}, \bar{g}\right)$ defined by $H=\frac{1}{n-1} \bar{g}^{i j} \bar{\omega}_{i j}$, and semicolon ";" indicates covariant differentiation. In particular, if $H=0$, then the totally umbilic hypersurface $\left(\bar{M}^{n-1}, \bar{g}\right)$ is called a totally geodesic hypersurface of $\left(M^{n}, g\right)$ [5]. The equations of Weingarten, Gauss and Codazzi for a totally umbilic hypersurface $\left(\bar{M}^{n-1}, \bar{g}\right)$ of $\left(M^{n}, g\right)$ are respectively obtained as

$$
\begin{gathered}
N_{; i}^{\alpha}=-H \frac{\partial y^{\alpha}}{\partial x^{i}}, \\
\bar{R}_{i j k l}=R_{\alpha \beta \gamma \delta} \frac{\partial y^{\alpha}}{\partial x^{i}} \frac{\partial y^{\beta}}{\partial x^{j}} \frac{\partial y^{\gamma}}{\partial x^{k}} \frac{\partial y^{\delta}}{\partial x^{l}}+H^{2}\left(\bar{g}_{i l} \bar{g}_{j k}-\bar{g}_{i k} \bar{g}_{j l}\right), \\
R_{\alpha \beta \gamma \delta} \frac{\partial y^{\alpha}}{\partial x^{i}} \frac{\partial y^{\beta}}{\partial x^{j}} \frac{\partial y^{\gamma}}{\partial x^{k}} N^{\delta}=H_{; i} \bar{g}_{j k}-H_{; j} \bar{g}_{i k} .
\end{gathered}
$$

\section{Hypersurfaces of $G R M_{n}$}

In this section we deal with some hypersurfaces of $G R M_{n}$. At first, concerning the covariant derivative of curvature tensor, we obtain 
Lemma 3.1. Let $\left(\bar{M}^{n-1}, \bar{g}\right)$ be a totally umbilic hypersurface of $\left(M^{n}, g\right)$. Then we have

$$
\begin{gathered}
\bar{R}_{i j k l ; p}=R_{\alpha \beta \gamma \delta ; \mu} \frac{\partial y^{\alpha}}{\partial x^{i}} \frac{\partial y^{\beta}}{\partial x^{j}} \frac{\partial y^{\gamma}}{\partial x^{k}} \frac{\partial y^{\delta}}{\partial x^{l}} \frac{\partial y^{\mu}}{\partial x^{p}}+ \\
+H H_{; i}\left(\bar{g}_{l p} \bar{g}_{j k}-\bar{g}_{k p} \bar{g}_{j l}\right)+H H_{; j}\left(\bar{g}_{i l} \bar{g}_{k p}-\bar{g}_{i k} \bar{g}_{l p}\right)+H H_{; k}\left(\bar{g}_{j p} \bar{g}_{l i}-\bar{g}_{i p} \bar{g}_{l j}\right)+ \\
+H H_{; l}\left(\bar{g}_{i p} \bar{g}_{k j}-\bar{g}_{j p} \bar{g}_{k i}\right)+2 H H_{; p}\left(\bar{g}_{i l} \bar{g}_{j k}-\bar{g}_{i k} \bar{g}_{j l}\right) \\
R_{\alpha \beta \gamma \delta ; \mu} \frac{\partial y^{\alpha}}{\partial x^{i}} \frac{\partial y^{\beta}}{\partial x^{j}} \frac{\partial y^{\gamma}}{\partial x^{k}} N^{\delta} \frac{\partial y^{\mu}}{\partial x^{p}}+H\left(R_{\alpha \beta \gamma \delta} N^{\alpha} \frac{\partial y^{\beta}}{\partial x^{j}} \frac{\partial y^{\gamma}}{\partial x^{k}} N^{\delta} \bar{g}_{i p}+\right. \\
+R_{\alpha \beta \gamma \delta} \frac{\partial y^{\alpha}}{\partial x^{i}} N^{\beta} \frac{\partial y^{\gamma}}{\partial x^{k}} N^{\delta} \bar{g}_{j p}+R_{\alpha \beta \gamma \delta} \frac{\partial y^{\alpha}}{\partial x^{i}} \frac{\partial y^{\beta}}{\partial x^{j}} N^{\gamma} N^{\delta} \bar{g}_{k p}+ \\
\left.-R_{\alpha \beta \gamma \delta} \frac{\partial y^{\alpha}}{\partial x^{i}} \frac{\partial y^{\beta}}{\partial x^{j}} \frac{\partial y^{\gamma}}{\partial x^{k}} \frac{\partial y^{\delta}}{\partial x^{p}}\right)=H_{; i p} \bar{g}_{j k}-H_{; j p} \bar{g}_{i k} .
\end{gathered}
$$

Proof. Differentiating (2.6) covariantly, we have

$$
\begin{gathered}
\bar{R}_{i j k l ; p}=R_{\alpha \beta \gamma \delta ; \mu} \frac{\partial y^{\mu}}{\partial x^{p}} \frac{\partial y^{\alpha}}{\partial x^{i}} \frac{\partial y^{\beta}}{\partial x^{j}} \frac{\partial y^{\gamma}}{\partial x^{k}} \frac{\partial y^{\delta}}{\partial x^{l}}+R_{\alpha \beta \gamma \delta}\left(\frac{\partial y^{\alpha}}{\partial x^{i}}\right)_{; p} \frac{\partial y^{\beta}}{\partial x^{j}} \frac{\partial y^{\gamma}}{\partial x^{k}} \frac{\partial y^{\delta}}{\partial x^{l}}+ \\
+R_{\alpha \beta \gamma \delta} \frac{\partial y^{\alpha}}{\partial x^{i}}\left(\frac{\partial y^{\beta}}{\partial x^{j}}\right)_{; p} \frac{\partial y^{\gamma}}{\partial x^{k}} \frac{\partial y^{\delta}}{\partial x^{l}}+R_{\alpha \beta \gamma \delta} \frac{\partial y^{\alpha}}{\partial x^{i}} \frac{\partial y^{\beta}}{\partial x^{j}}\left(\frac{\partial y^{\gamma}}{\partial x^{k}}\right)_{; p} \frac{\partial y^{\delta}}{\partial x^{l}}+ \\
+R_{\alpha \beta \gamma \delta} \frac{\partial y^{\alpha}}{\partial x^{i}} \frac{\partial y^{\beta}}{\partial x^{j}} \frac{\partial y^{\gamma}}{\partial x^{k}}\left(\frac{\partial y^{\delta}}{\partial x^{l}}\right)_{; p}+2 H H_{; p}\left(\bar{g}_{i l} \bar{g}_{j k}-\bar{g}_{i k} \bar{g}_{j l}\right) .
\end{gathered}
$$

By virtue of (2.4) and the last relation, we obtain

$$
\begin{gathered}
\bar{R}_{i j k l ; p}=R_{\alpha \beta \gamma \delta ; \mu} \frac{\partial y^{\mu}}{\partial x^{p}} \frac{\partial y^{\alpha}}{\partial x^{i}} \frac{\partial y^{\beta}}{\partial x^{j}} \frac{\partial y^{\gamma}}{\partial x^{k}} \frac{\partial y^{\delta}}{\partial x^{l}}+\bar{g}_{i p} H R_{\alpha \beta \gamma \delta} N^{\alpha} \frac{\partial y^{\beta}}{\partial x^{j}} \frac{\partial y^{\gamma}}{\partial x^{k}} \frac{\partial y^{\delta}}{\partial x^{l}}+ \\
+\bar{g}_{j p} H R_{\alpha \beta \gamma \delta} \frac{\partial y^{\alpha}}{\partial x^{i}} N^{\beta} \frac{\partial y^{\gamma}}{\partial x^{k}} \frac{\partial y^{\delta}}{\partial x^{l}}+\bar{g}_{k p} H R_{\alpha \beta \gamma \delta} \frac{\partial y^{\alpha}}{\partial x^{i}} \frac{\partial y^{\beta}}{\partial x^{j}} N^{\gamma} \frac{\partial y^{\delta}}{\partial x^{l}}+ \\
+\bar{g}_{l p} H R_{\alpha \beta \gamma \delta} \frac{\partial y^{\alpha}}{\partial x^{i}} \frac{\partial y^{\beta}}{\partial x^{j}} \frac{\partial y^{\gamma}}{\partial x^{k}} N^{\delta}+2 H H_{; p}\left(\bar{g}_{i l} \bar{g}_{j k}-\bar{g}_{i k} \bar{g}_{j l}\right) .
\end{gathered}
$$

It follows from (2.7) that the last relation reduces to

$$
\begin{gathered}
\bar{R}_{i j k l ; p}=R_{\alpha \beta \gamma \delta ; \mu} \frac{\partial y^{\alpha}}{\partial x^{i}} \frac{\partial y^{\beta}}{\partial x^{j}} \frac{\partial y^{\gamma}}{\partial x^{k}} \frac{\partial y^{\delta}}{\partial x^{l}} \frac{\partial y^{\mu}}{\partial x^{p}}+ \\
+H H_{; i}\left(\bar{g}_{l p} \bar{g}_{j k}-\bar{g}_{k p} \bar{g}_{j l}\right)+H H_{; j}\left(\bar{g}_{i l} \bar{g}_{k p}-\bar{g}_{i k} \bar{g}_{l p}\right)+H H_{; k}\left(\bar{g}_{j p} \bar{g}_{l i}-\bar{g}_{i p} \bar{g}_{l j}\right)+ \\
+H H_{; l}\left(\bar{g}_{i p} \bar{g}_{k j}-\bar{g}_{j p} \bar{g}_{k i}\right)+2 H H_{; p}\left(\bar{g}_{i l} \bar{g}_{j k}-\bar{g}_{i k} \bar{g}_{j l}\right) .
\end{gathered}
$$


On the other hand, differentiating (2.7) covariantly, we get

$$
\begin{aligned}
& R_{\alpha \beta \gamma \delta ; \mu} \frac{\partial y^{\mu}}{\partial x^{p}} \frac{\partial y^{\alpha}}{\partial x^{i}} \frac{\partial y^{\beta}}{\partial x^{j}} \frac{\partial y^{\gamma}}{\partial x^{k}} N^{\delta} \\
& +R_{\alpha \beta \gamma \delta}\left(\frac{\partial y^{\alpha}}{\partial x^{i}}\right)_{; p} \frac{\partial y^{\beta}}{\partial x^{j}} \frac{\partial y^{\gamma}}{\partial x^{k}} N^{\delta}+R_{\alpha \beta \gamma \delta} \frac{\partial y^{\alpha}}{\partial x^{i}}\left(\frac{\partial y^{\beta}}{\partial x^{j}}\right)_{; p} \frac{\partial y^{\gamma}}{\partial x^{k}} N^{\delta} \\
& +R_{\alpha \beta \gamma \delta} \frac{\partial y^{\alpha}}{\partial x^{i}} \frac{\partial y^{\beta}}{\partial x^{j}}\left(\frac{\partial y^{\gamma}}{\partial x^{k}}\right)_{; p} N^{\delta}+R_{\alpha \beta \gamma \delta} \frac{\partial y^{\alpha}}{\partial x^{i}} \frac{\partial y^{\beta}}{\partial x^{j}} \frac{\partial y^{\gamma}}{\partial x^{k}} N_{; p}^{\delta} \\
& =H_{; i p} \bar{g}_{j k}-H_{; j p} \bar{g}_{i k} .
\end{aligned}
$$

Taking account of (2.4), (2.5) and the last relation, we have

$$
\begin{aligned}
& R_{\alpha \beta \gamma \delta ; \mu} \frac{\partial y^{\alpha}}{\partial x^{i}} \frac{\partial y^{\beta}}{\partial x^{j}} \frac{\partial y^{\gamma}}{\partial x^{k}} N^{\delta} \frac{\partial y^{\mu}}{\partial x^{p}} \\
& +H\left(R_{\alpha \beta \gamma \delta} N^{\alpha} \frac{\partial y^{\beta}}{\partial x^{j}} \frac{\partial y^{\gamma}}{\partial x^{k}} N^{\delta} \bar{g}_{i p}+R_{\alpha \beta \gamma \delta} \frac{\partial y^{\alpha}}{\partial x^{i}} N^{\beta} \frac{\partial y^{\gamma}}{\partial x^{k}} N^{\delta} \bar{g}_{j p}\right. \\
& \left.+R_{\alpha \beta \gamma \delta} \frac{\partial y^{\alpha}}{\partial x^{i}} \frac{\partial y^{\beta}}{\partial x^{j}} N^{\gamma} N^{\delta} \bar{g}_{k p}-R_{\alpha \beta \gamma \delta} \frac{\partial y^{\alpha}}{\partial x^{i}} \frac{\partial y^{\beta}}{\partial x^{j}} \frac{\partial y^{\gamma}}{\partial x^{k}} \frac{\partial y^{\delta}}{\partial x^{p}}\right) \\
& =H_{; i p} \bar{g}_{j k}-H_{; j p} \bar{g}_{i k} .
\end{aligned}
$$

This completes the proof.

Theorem 3.2. Let $\left(M^{n}, g\right)$ be a $G R M_{n}$. If $\left(\bar{M}^{n-1}, \bar{g}\right)$ is a totally geodesic hypersurface of $\left(M^{n}, g\right)$, then the manifold $\left(\bar{M}^{n-1}, \bar{g}\right)$ is a $G R M_{n-1}$.

Proof. By virtue of $H=0$, we have from (3.8)

$$
\bar{R}_{i j k l ; p}=R_{\alpha \beta \gamma \delta ; \mu} \frac{\partial y^{\alpha}}{\partial x^{i}} \frac{\partial y^{\beta}}{\partial x^{j}} \frac{\partial y^{\gamma}}{\partial x^{k}} \frac{\partial y^{\delta}}{\partial x^{l}} \frac{\partial y^{\mu}}{\partial x^{p}} .
$$

Since $\left(M^{n}, g\right)$ is a $G R M_{n}$, the last relation yields from (1.1)

$$
\bar{R}_{i j k l ; p}=A_{\mu} R_{\alpha \beta \gamma \delta} \frac{\partial y^{\alpha}}{\partial x^{i}} \frac{\partial y^{\beta}}{\partial x^{j}} \frac{\partial y^{\gamma}}{\partial x^{k}} \frac{\partial y^{\delta}}{\partial x^{l}} \frac{\partial y^{\mu}}{\partial x^{p}}+B_{\mu}(g \bullet g)_{\alpha \beta \gamma \delta} \frac{\partial y^{\alpha}}{\partial x^{i}} \frac{\partial y^{\beta}}{\partial x^{j}} \frac{\partial y^{\gamma}}{\partial x^{k}} \frac{\partial y^{\delta}}{\partial x^{l}} \frac{\partial y^{\mu}}{\partial x^{p}}
$$

Because of (2.6) and $H=0$, the last relation reduces to

$$
\bar{R}_{i j k l ; p}=A_{p} \bar{R}_{i j k l}+B_{p}(\bar{g} \bullet \bar{g})_{i j k l},
$$

showing that the manifold is a $G R M_{n-1}$. This completes the proof. 


\section{Riemannian Product $G R M_{n}$}

Let $\left(M^{n}, g\right)$ be a Riemannian product manifold $\left(M^{p} \times M^{n-p}, \widehat{g}+\widetilde{g}\right)$. In local coordinates, we adopt the Latin indices (resp. the Greek indices) for tensor components which are constructed on $\left(M^{p}, \widehat{g}\right)\left(\operatorname{resp} .\left(M^{n-p}, \widetilde{g}\right)\right)$. Therefore, the Latin indices take the values from $1, \ldots, p$ whereas the Greek indices run over the range $p+1, \ldots, n$. Now we can state the followings.

Theorem 4.1. Let a Riemannian product manifold $\left(M^{p} \times M^{n-p}, \widehat{g}+\widetilde{g}\right)$ be a $G R M_{n}$. Then either one decomposition manifold $\left(M^{p}, \widehat{g}\right)$ is locally symmetric or the other decomposition manifold $\left(M^{n-p}, \widetilde{g}\right)$ is a space of constant curvature.

Proof. Since any tensor components of $R$ and its covariant derivatives with both Latin and Greek indices together should be zero, we have from (1.1) and $R_{\alpha \beta \gamma \delta ; p}=0$

$$
0=A_{p} R_{\alpha \beta \gamma \delta}+B_{p}(g \bullet g)_{\alpha \beta \gamma \delta} .
$$

If we assume that $A_{p}=0$, then from (4.10) and $(g \bullet g)_{\alpha \beta \gamma \delta} \neq 0$ it follows that $B_{p}=0$, which yields from (1.1)

$$
R_{i j k l ; p}=0
$$

showing that $\left(M^{p}, \widehat{g}\right)$ is locally symmetric.

On the other hand, if we assume that $A_{p} \neq 0$, then it follows from (4.10) that

$$
R_{\alpha \beta \gamma \delta}=-\frac{B_{p}}{A_{p}}(g \bullet g)_{\alpha \beta \gamma \delta},
$$

showing that $\left(M^{n-p}, \widetilde{g}\right)$ is a space of constant curvature. This completes the proof.

Theorem 4.2. Let $\left(M^{n}, g_{c}\right)$ be a space of constant curvature. Then the Riemannian product manifold $\left(M^{2 n}, g\right)$ of $\left(M^{n}, g_{c}\right)$ with itself is a $G R M_{2 n}$.

Proof. Since $\left(M^{n}, g_{c}\right)$ is a space of constant curvature, we have

$$
R_{i j k l}^{c}=\frac{s}{2 n(n-1)}\left(g_{c} \bullet g_{c}\right)_{i j k l}
$$

and

$$
R_{i j k l ; p}^{c}=0 .
$$


Here $R^{c}$ and $s$ denote the curvature tensor and the scalar curvature respectively on $\left(M^{n}, g_{c}\right)$. Therefore, from (4.13) and (4.14) it follows that

$$
R_{i j k l ; p}^{c}=R_{i j k l}^{c}-\frac{s}{2 n(n-1)}\left(g_{c} \bullet g_{c}\right)_{i j k l} .
$$

Now we consider the Riemannian product manifold $\left(M^{2 n}, g\right)$ of a space of constant curvature $\left(M^{n}, g_{c}\right)$ with itself. Obviously the Riemannian curvature tensor $R$ of $\left(M^{2 n}, g\right)$ satisfies

$$
R_{i j k l}=R_{i j k l}^{c}+R_{i j k l}^{c}
$$

and

$$
R_{i j k l ; p}=0=R_{i j k l ; p}^{c}+R_{i j k l ; p}^{c},
$$

which yields from (4.15) and the last relations

$$
R_{i j k l ; p}=R_{i j k l}-\frac{s}{2 n(n-1)}\left(\frac{1}{2}\right)(g \bullet g)_{i j k l}
$$

because of

$$
\left(g_{c} \bullet g_{c}\right)+\left(g_{c} \bullet g_{c}\right)=\frac{1}{2}\left(g_{c}+g_{c}\right) \bullet\left(g_{c}+g_{c}\right)=\frac{1}{2} g \bullet g .
$$

Therefore we have

$$
R_{i j k l ; p}=A_{p} R_{i j k l}+B_{p}(g \bullet g)_{i j k l},
$$

where $A_{p}=1$ and $B_{p}=-\frac{s}{4 n(n-1)}$, showing that the Riemannian product manifold $\left(M^{2 n}, g\right)$ of a space of constant curvature $\left(M^{n}, g_{c}\right)$ with itself is a $G R M_{2 n}$. This completes the proof.

\section{References}

[1] T. Adati, T. Miyazawa, On Riemannian space with recurrent conformal curvature, Tensor (N.S.) 18 (1967), 348-354.

[2] T. Adati, T. Miyazawa, On projective transformations of projective recurrent spaces, Tensor (N.S.) 31 (1977), 49-54.

[3] K. Arslan, U.C. De, C. Murathan, A. Yildiz, On generalized recurrent Riemannian manifolds, Acta Math.Hungar. 123 (2009), 27-39. 
[4] E. Cartan, Sur une classe remarquable despaces de Riemann. I, Bull.de la Soc.Math.de France 54 (1926), 214-216.

[5] B.Y. Chen, Geometry of submanifolds, Marcel-Deker, New York (1973).

[6] R.S.D. Dubey, Generalized recurrent spaces, Indian J.Pure Appl.Math. 10 (1979), 1508-1513.

[7] A. Lichnerowicz, Courbure, nombres de Betti, et espaces symmetriques, Proc.Int.Cong.Math. 2 (1952), 216-223.

[8] T. Miyazawa, On Riemannian space admitting some recurrent tensor, TRU Math.J. 2 (1996), 11-18.

[9] E.M. Patterson, Some theorems on Ricci recurrent spaces, J.Lond.Math.Soc. 27 (1952), 287-295.

[10] A.G. Walker, On Ruse's spaces of recurrent curvature, Proc.London Math.Soc. 52 (1950), 36-64. 